\title{
Carbon C 14 Vemurafenib
}

National Cancer Institute

\section{Source}

National Cancer Institute. Carbon C 14 Vemurafenib. NCI Thesaurus. Code C121958.

A radioconjug ate composed of vemurafenib, an AT P-competitive, small-molecule inhibitor of BRAF(V600E) kinase, labeled with the radioisotope carbon C 14, with potential use for evaluating the pharmacokinetic profile of vemurafenib. Vemurafenib selectively binds to the ATP-binding site of BRAF(V600E) kinase and inhibits its activity, which may result in both an inhibition of an over-activated MAPK signaling pathway in BRAF(V600E) kinase-expressing tumor cells and a reduction in tumor cell proliferation. Labeling of vemurafenib with the radioactive tracer carbon C 14 allows for the evaluation of vemurafenib's pharmacokinetic profile, including its absorption, distribution, metabolism, and excretion (ADME). The BRAF(V600E) gene mutation in which valine is substituted for glutamic acid at residue 600 (V600E), is found in many cancer cell types and plays a key role in the over-activation of the MAPK signaling pathway. 